\title{
Cardioprotective medication adherence among patients with coronary heart disease in China: a systematic review
}

\author{
Zhao $\mathrm{Ni}_{,}{ }^{1}$ Latefa Dardas, $^{\oplus 2}{ }^{2}$ Bei Wu, ${ }^{3}$ Ryan Shaw ${ }^{1}$
}

${ }^{1}$ School of Nursing, Duke University, Durham, North Carolina, USA

${ }^{2}$ School of Nursing, The University of Jordan, Amman, Jordan

${ }^{3}$ Rory Meyers College of Nursing, New York University, New York City, New York, USA

\section{Correspondence to}

Dr Zhao Ni, School of Nursing, Duke University, Durham, North Carolina, USA; zhao.ni@alumni. duke.edu

Received 27 December 2018

Revised 2 May 2019

Accepted 21 May 2019
Check for updates

(C) Author(s) (or their employer(s)) 2019. No commercial re-use. See rights and permissions. Published by BMJ.

To cite: Ni Z, Dardas L,

Wu B, et al. Heart Asia

2019;11:e011173.

doi:10.1136/

heartasia-2018-011173

\section{ABSTRACT}

In China, poor cardioprotective medication adherence is a key reason for the high mortality rate of coronary heart disease (CHD). The aims of this systematic review are to (1) describe and synthesise factors that influence medication adherence among Chinese people with CHD, (2) evaluate the current status of intervention studies, and (3) discuss directions of future research to improve medication adherence. A comprehensive search using PubMed, Cumulative Index to Nursing and Allied Health Literature, Embase, Scopus, Global Health and PsycINFO was undertaken to describe poor adherence in China. Thirty-three eligible articles were included in the study. The review shows that there are multiple contributing factors to poor medication adherence, including patients' sociodemographic characteristics, health status and medication characteristics. In addition, from patients' perspective, lack of medication-related knowledge, such as the name, function, dosage and frequency, contributes to poor adherence. From physicians' perspective, a gap exists between CHD secondary prevention guidelines and clinical practice in China. Follow-up phone calls, educational lectures, booklets and reminder cards were common methods found to be effective in improving medication adherence. This systematic review indicates that cardioprotective medications were commonly prescribed as secondary prevention medication to patients with CHD in China, but adherence to these medications gradually decreased during a follow-up period. Therefore, more research should be conducted on how to establish highquality health educational programmes aimed at increasing patients' medication adherence.

\section{INTRODUCTION}

Poor medication adherence among patients with coronary heart disease (CHD) is a global public health concern. ${ }^{1}$ This is particularly the case in China $^{23}$ given the large size of patient population and the significant health disparities in access to care across its regions. ${ }^{4} \mathrm{CHD}$ is the second leading cause of death in China, ${ }^{5}{ }^{6}$ leading to over 1.5 million deaths each year. ${ }^{5}$ Cardioprotective medications, including antiplatelet drugs, beta-blockers, calcium channel blockers, statins and ACE inhibitors, are an essential treatment modality for $\mathrm{CHD}^{7}$ and can significantly reduce the mortality rate of this disease. ${ }^{3-12}$ However, poor adherence to cardioprotective medications is a public health threat in China. ${ }^{2} 13$ Many previous studies highlighted the importance of medication adherence in preventing cardiovascular diseases and the factors related to medication non-adherence, but none of them systematically summarised the factors among
Chinese patients with CHD. Moreover, in China, there is a significant gap between factors found in the literature that related to medication non-adherence and interventions taken to tackle the problem in practice. Many previous studies investigated the factors that contributed to medication non-adherence, but did not propose specific interventions to improve medication adherence. A deeper understanding of the interplay of factors related to adherence to cardioprotective medications among the Chinese population is needed in order to develop interventions that will appropriately target this phenomenon. ${ }^{14}{ }^{15}$ Therefore, the aims of this systematic review are to (1) describe and synthesise factors that influence medication adherence among Chinese people with CHD, (2) evaluate the current status of intervention studies, and (3) discuss directions of future research to improve medication adherence. The findings from this review may provide insights to address the issue of medication non-adherence globally.

\section{METHODS}

\section{Search strategy}

We chose the databases PubMed, Cumulative Index to Nursing and Allied Health Literature (CINAHL), Embase, Scopus, Global Health and PsycINFO as primary data sources for this review, as together they provide a comprehensive coverage of research in health-related disciplines. Two authors (ZN and LD) searched each database using keywords and algorithms that included the following: (coronary heart disease OR coronary artery disease), and (China OR Chinese), and (adherence OR compliance OR persistence OR discontinue OR underuse OR non-adherence OR non-compliance OR comply OR compliant OR adhere). An academic health centre reference librarian provided key assistance in building the combination of index and search terms that were applied to each database (table 1 ). No restrictions were applied on publication date. The first author $(\mathrm{ZN})$, who is a native Chinese speaker, ran another search within a Chinese database, the China National Knowledge Infrastructure (CNKI), and used the Chinese keywords 'yao wu yi cong xing' (药物依从 性) and 'guan xin bing' (冠心病), meaning medication adherence and coronary heart disease.

\section{Inclusion and exclusion criteria}

We included articles that met the following criteria: (1) focus on adherence to at least one of the following cardioprotective medications: antiplatelet drugs, beta-blockers, calcium channel blockers, statins and 
Table 1 Search trial on PubMed, CINAHL, Embase, Scopus, Global Health and PsycINFO

\begin{tabular}{|c|c|c|c|}
\hline Database & $\begin{array}{l}\text { Search } \\
\text { number }\end{array}$ & Medical Subject Headings (MeSH) terms and keywords & $\begin{array}{l}\text { Articles } \\
\text { revealed }\end{array}$ \\
\hline \multirow[t]{4}{*}{ PubMed } & $\# 1$ & $\begin{array}{l}\text { ("coronary disease" [MeSH Terms] OR ("coronary" [All Fields] AND "disease" [All Fields]) OR "coronary disease" [All Fields] OR ("coronary" [All } \\
\text { Fields] AND "heart" [All Fields] AND "disease" [All Fields]) OR "coronary heart disease" [All Fields]) OR ("coronary artery disease" [MeSH Terms] } \\
\text { OR ("coronary" [All Fields] AND "artery" [All Fields] AND "disease" [All Fields]) OR "coronary artery disease" [All Fields)) }\end{array}$ & 294895 \\
\hline & \#2 & $\begin{array}{l}\text { "china" [MeSH Terms] OR "china" [All Fields)) OR ("asian continental ancestry group" [MeSH Terms] OR ("asian" [All Fields] AND "continental" } \\
\text { [All Fields] AND "ancestry" [All Fields] AND "group" [All Fields]) OR "asian continental ancestry group" [All Fields] OR "chinese" [All Fields]) }\end{array}$ & 1357102 \\
\hline & \#3 & $\begin{array}{l}\text { adherence [All Fields] OR ("patient compliance" [MeSH Terms] OR ("patient" [All Fields] AND "compliance" [All Fields]) OR "patient compliance" } \\
\text { [All Fields] OR "compliance" [All Fields] OR "compliance" [MeSH Terms]) OR persistence [All Fields] OR discontinue [All Fields] OR underuse [All } \\
\text { Fields] OR non-adherence [All Fields] OR non-compliance [All Fields] OR comply [All Fields] OR ("patient compliance" [MeSH Terms] OR ("patient" } \\
\text { [All Fields] AND "compliance" [All Fields]) OR "patient compliance" [All Fields] OR "compliant" [All Fields]) OR adhere [All Fields] }\end{array}$ & 353524 \\
\hline & \#4 & \#1 AND \#2 AND \#3 & 173 \\
\hline \multirow[t]{4}{*}{ CINAHL } & $\# 1$ & (coronary heart disease OR coronary artery disease) & 33626 \\
\hline & \#2 & (China OR Chinese) & 59960 \\
\hline & \#3 & $\begin{array}{l}\text { (adherence OR compliance OR persistence OR discontinue OR underuse OR non-adherence OR non-compliance OR comply OR compliant OR } \\
\text { adhere) }\end{array}$ & 105750 \\
\hline & \#4 & \#1 AND \#2 AND \#3 & 23 \\
\hline \multirow[t]{4}{*}{ Embase } & $\# 1$ & (coronary AND ('heart'/exp OR heart) AND ('disease'/exp OR disease) OR coronary) AND ('artery'/exp OR artery) AND ('disease'/exp OR disease) & 433989 \\
\hline & \#2 & china OR chinese & 1695781 \\
\hline & \#3 & $\begin{array}{l}\text { adherence OR compliance OR persistence OR discontinue OR underuse OR 'non adherence' OR 'non-compliance' OR comply OR compliant OR } \\
\text { adhere }\end{array}$ & 524776 \\
\hline & \#4 & \#1 AND \#2 AND \#3 & 320 \\
\hline \multirow[t]{4}{*}{ Scopus } & \#1 & (coronary heart disease OR coronary artery disease) & 201258 \\
\hline & \#2 & (China OR Chinese) & 1096428 \\
\hline & \#3 & $\begin{array}{l}\text { (adherence OR compliance OR persistence OR discontinue OR underuse OR non-adherence OR non-compliance OR comply OR compliant OR } \\
\text { adhere) }\end{array}$ & 696604 \\
\hline & \#4 & \#1 AND \#2 AND \#3 & 54 \\
\hline \multirow{4}{*}{$\begin{array}{l}\text { Global } \\
\text { Health }\end{array}$} & $\# 1$ & (coronary heart disease OR coronary artery disease) & 39659 \\
\hline & \#2 & (China OR Chinese) & 315129 \\
\hline & \#3 & $\begin{array}{l}\text { (adherence OR compliance OR persistence OR discontinue OR underuse OR non-adherence OR non-compliance OR comply OR compliant OR } \\
\text { adhere) }\end{array}$ & 58414 \\
\hline & \#4 & \#1 AND \#2 AND \#3 & 56 \\
\hline \multirow[t]{4}{*}{ PsycINFO } & $\# 1$ & (coronary heart disease OR coronary artery disease) & 6844 \\
\hline & \#2 & (China OR Chinese) & 99722 \\
\hline & \#3 & $\begin{array}{l}\text { (adherence OR compliance OR persistence OR discontinue OR underuse OR non-adherence OR non-compliance OR comply OR compliant OR } \\
\text { adhere) }\end{array}$ & 80844 \\
\hline & \#4 & \#1 AND \#2 AND \#3 & 8 \\
\hline
\end{tabular}

CINAHL, Cumulative Index to Nursing and Allied Health Literature.

ACE inhibitors/angiotensin receptor blocker; (2) Chinese samples with CHD living in China; and (3) peer-reviewed articles published in English or Chinese. Studies that included samples of Chinese patients living in Hong Kong, Taiwan and Macao were excluded, as these areas are historically dominated by foreign cultures, and thus their social structures and healthcare systems are different from that in mainland China.

\section{Search outcomes}

Our search resulted in the following: 173 articles from PubMed, 23 from CINAHL, 320 from Embase, 54 from Scopus, 56 from Global Health and 8 from PsycINFO. From the resulting 634 articles, 168 duplicates were removed, yielding 466 results. Afterwards, the first and the second authors (ZN and LD) screened the 466 titles independently and compared and discussed their results until agreement was reached, yielding 134 articles for subsequent abstract screening. After evaluating the abstracts of the 134 articles, 80 articles were excluded based on the preset inclusion criteria. The full texts of the remaining 54 articles were then examined and 26 articles met the full inclusion criteria (figure 1). The search of Chinese literature in CNKI yielded 100 related articles. After screening the titles, abstracts and full texts, seven articles were included (figure 2). Together, a total of 33 articles were used in this study. Later, we used a set of criteria to assess the quality of evidence provided by each of the included 33 studies. These criteria included study design, sample selection, measures, statistical analyses, reporting, content and utility (table 2).

\section{FINDINGS}

\section{Study characteristics}

The 33 articles included in this systematic review came from the medical, nursing and pharmacological disciplines. A range of study designs were employed from cross-sectional studies $(n=22)$ and longitudinal studies $(n=2)$, to randomised controlled trials $(n=9)$ (table 3). In total, 52008 participants were included in this systematic review, with only 18278 (35.14\%) women. Importantly, participants were from over 508 medical institutions located in over 17 Chinese provinces (out of a total of 34 provincial-level administrative units), with almost all studies conducted in hospitals located in urban areas of China. Only two studies were conducted in rural areas. There was significant heterogeneity between the studies in terms of instruments used to measure medication adherence, making a robust meta-analysis or statistical analysis difficult to conduct. Thus, a qualitative summary of the findings is presented. We organised our findings with three core themes: (1) Lack of knowledge and follow-up care are barriers to medication adherence; (2) patients' sociodemographic characteristics, health status and medication characteristics influencing adherence; and (3) 


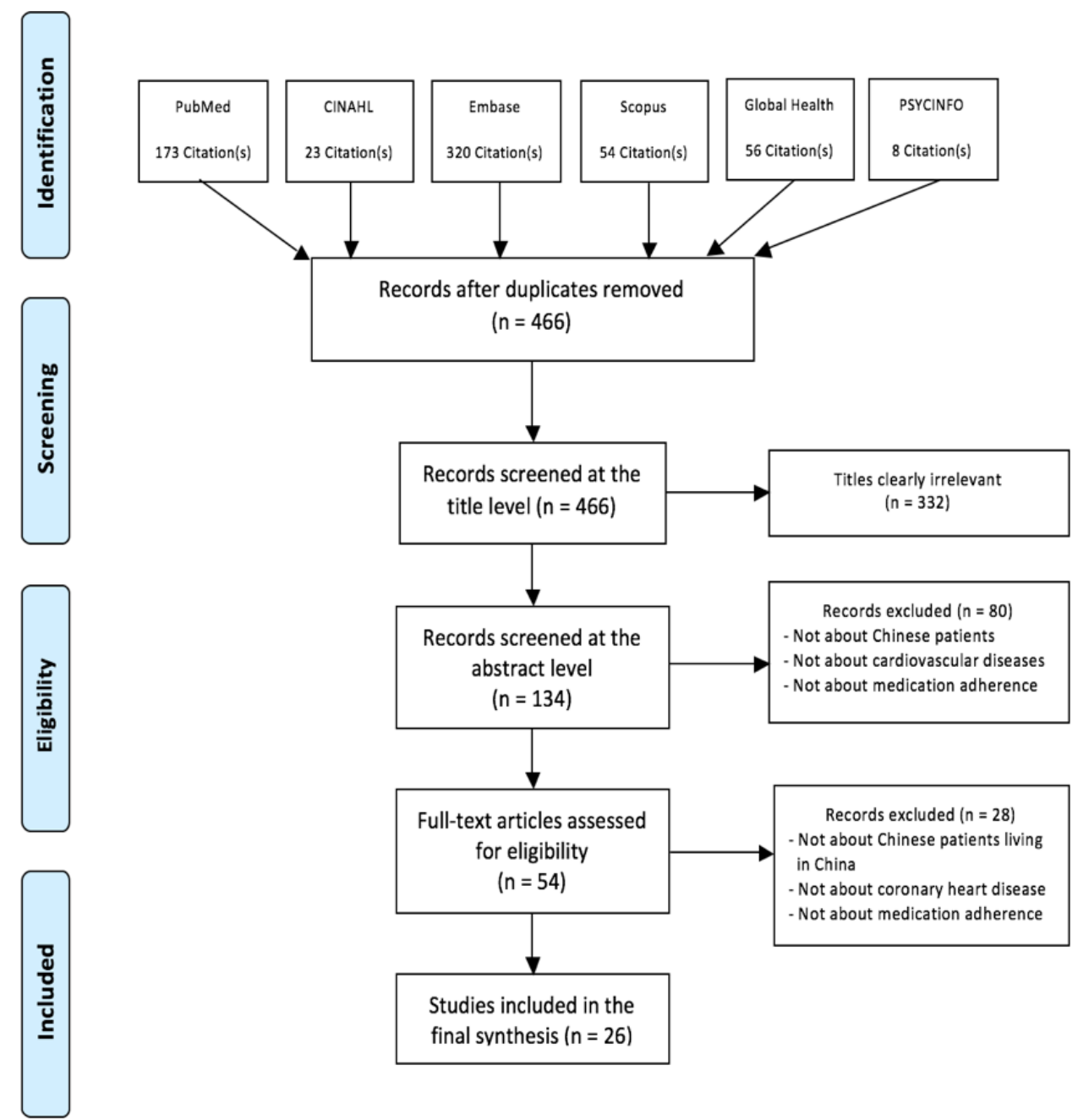

Figure 1 Literature review flow chart for English database. Adapted from the Preferred Reporting Items for Systematic Reviews and Meta-Analyses 2009 flow diagram. ${ }^{50}$ CINAHL, Cumulative Index to Nursing and Allied Health Literature.

various intervention methods used for improvement of medication adherence. Subthemes are presented within each of these three core themes.

\section{Lack of knowledge and follow-up care are barriers to medication adherence (12 articles)}

Patients' lack of knowledge (five articles)

Our systematic review revealed that patients' lack of knowledge about their medications was a substantial barrier to adherence. For example, Wang and $\mathrm{Li}^{16}$ investigated the adherence to statins among 1368 elderly patients with CHD over 80 years of age, and found that lack of knowledge of CHD and statins was the main factor influencing their adherence to statins. Participants did not understand the importance of taking medications over the long term, did not know the morbidities of CHD and did not understand that statins can prevent CHD. ${ }^{16}$ Similarly, $\mathrm{Li}^{17}$ found that lack of knowledge of CHD was significantly related to poor adherence to statins among patients. Lack of knowledge was also a barrier to adherence among patients with uncontrolled cholesterol levels. Ding et $a l^{18}$ found that of 903 patients with CHD, 18.2\% did not take statins. One major reason for those patients to not take statins was because they lack knowledge related to statins; they did not know that they should routinely control their low-density lipoprotein-cholesterol level by statins. Finally, Zhao et $a l^{19}$ conducted a descriptive correlational study among 159 participants with CHD and revealed that up to $38 \%$ of the participants were non-adherent and that lack of knowledge about medication and disease was significantly associated with non-adherence. Patients with good knowledge of the medication and disease were more likely to adhere to medication instructions. ${ }^{19}$ Dai $^{20}$ surveyed 200 patients with CHD living in a Chinese rural area and found that medication adherence was low among those patients living in rural China, with only $6.4 \%$ of the participants adhering to their cardioprotective medications. Factors that contributed to poor medication adherence included poor communication between healthcare providers and patients, healthcare providers not fully explaining CHD and medications, and patients' lack of clarity on factors to pay attention to while taking medications. ${ }^{20}$

Physicians' practice and the lack of follow-up care regarding medication-taking behaviours (nine articles)

In China, there is a lack of proper follow-up care regarding medication-taking behaviours among patients with CHD. Bi et al ${ }^{2}$ investigated 2973 patients with acute coronary syndromes in 49 hospitals in China on their medication-taking behaviour at the time of hospitalisation and one year after hospital discharge. Bi et $a l^{2}$ found that sustained use of medications among participants was suboptimal; the use of recommended cardioprotective medications continued to decrease over time. Eighty per cent of non-adherent participants reported that they did not take antiplatelets after discharge because they did not receive follow-up information from physicians that they need to continue to take medications. ${ }^{2}$ Similarly, $\mathrm{Hu}$ et $a l^{21}$ 


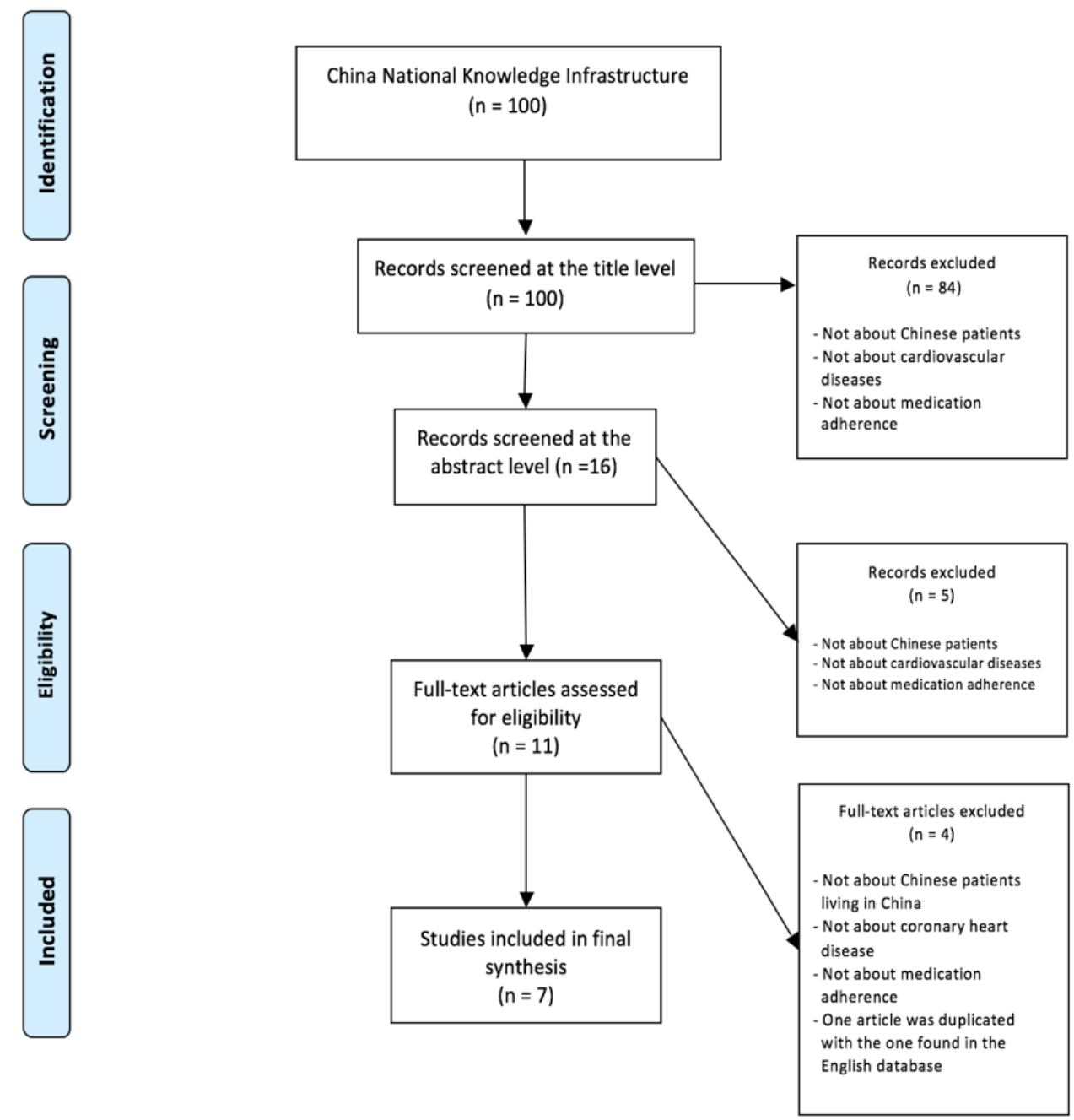

Figure 2 Literature review flow chart for Chinese database. Adapted from the Preferred Reporting Items for Systematic Reviews and Meta-Analyses 2009 flow diagram. ${ }^{50}$

and Li et $a^{22}$ conducted large multicentre studies surveying statin usage in outpatients with CHD and found that, although statins were prescribed for outpatients by physicians, the rate of achieving the target cholesterol level was low. The possible reason for this suboptimal health outcome was lack of follow-up care for patients.

Zhang et $\mathrm{al}^{12}$ studied the long-term beta-blocker therapy after hospital discharge among patients with CHD and found that only $49 \%$ participants consistently used beta-blocker after discharge and 22\% participants never used it, although consistent use of beta-blocker can lower mortality and cardiovascular events. Base on this finding, Zhang $e t a l^{12}$ recommended that improving long-term medication adherence among patients with CHD is necessary, and to improve this long-term adherence actions should be taken to promote patients' follow-up care regarding medication-taking behaviours and to improve physicians' prescribing practices. In more recent studies, Wang et $a l^{23}$ and Li et $a l^{24}$ found that physicians' prescribing practices could influence patients' medication-taking behaviours and health outcomes. The results from Jiang et al ${ }^{13}$ supported these findings. Jiang et al ${ }^{13}$ studied 837 physicians from cardiology departments in 35 tertiary hospitals in China and found that a gap between guideline requirements (American Heart Association/American College of Cardiology Guidelines for Secondary Prevention for Patients with Coronary and Other Atherosclerotic Vascular Disease) and clinical practice in CHD secondary prevention contributed to poor medication adherence in China. Likewise, Ding et $a l^{18}$ and Wang et $a l^{23}$ found that a disparity between guidelines and physicians' prescribing practices for patients with CHD exists in China. Xu et al's ${ }^{25}$ study showed that physicians from traditional Chinese medicine hospitals were less likely to prescribe beta-blockers and ACE inhibitors for patients with CHD in comparison with physicians from West medicine hospitals in China. Overall, our review showed that physicians' prescribing practices can influence patients' medication-taking behaviours, and lack of follow-up care for patients with CHD regarding medication-taking behaviours was a consistent barrier to medication adherence among Chinese patients with CHD. ${ }^{122-25}$

Patients' sociodemographic characteristics, health status and medication characteristics influencing adherence

Sociodemographic characteristics that are associated with adherence

Age (five articles)

Several studies 316172627 have demonstrated a link between older adults and poor adherence to cardioprotective medication. For example, Jin $e t a l^{3}$ found that among Chinese patients with acute coronary syndrome, medication non-adherence was common among older patients aged 65 and above. Li et $a l^{26}$ also found that adherence to statins was low among older adults. To further analyse adherence to statins in elderly patients, Wang and $\mathrm{Li}^{16}$ chose 1368 elderly patients aged over 80 years with CHD and 
Table 2 Quality appraisal of included studies

\begin{tabular}{|c|c|c|c|c|c|c|}
\hline $\begin{array}{l}\text { Author, } \\
\text { year }\end{array}$ & Study design & Inclusion criteria & Validated measures & Data analysis & Reporting & Content and utility \\
\hline Jin et al, $2014^{3}$ & $\mathrm{~N}$ & Y & $\mathrm{N}$ & Y & Y & Y \\
\hline Jiang et al, $2012^{13}$ & $\mathrm{~N}$ & $\mathrm{~N}$ & NA & Y & Y & Y \\
\hline Wang and $L i, 2014^{16}$ & $\mathrm{~N}$ & $\mathrm{~N}$ & Y & $\mathrm{N}$ & Y & Y \\
\hline Zhao et al, $2015^{19}$ & $\mathrm{~N}$ & Y & Y & $\mathrm{N}$ & Y & Y \\
\hline Dai, $2013^{20}$ & Y & Y & $\mathrm{N}$ & $\mathrm{N}$ & N & N \\
\hline Hu et al, $2008^{21}$ & $\mathrm{~N}$ & $\mathrm{~N}$ & $\mathrm{~N}$ & Y & Y & Y \\
\hline Jiang et al, $2015^{29}$ & Y & Y & $\mathrm{N}$ & Y & Y & Y \\
\hline Xu et al, $2012^{25}$ & $\mathrm{Y}$ & Y & $\mathrm{N}$ & Y & Y & Y \\
\hline Zhuang et al, $2016^{27}$ & Y & Y & N & Y & Y & Y \\
\hline Yang et al, $2012^{28}$ & Y & Y & Y & $\mathrm{N}$ & Y & Y \\
\hline Huang et al, $2014^{30}$ & $\mathrm{~N}$ & Y & Y & $\mathrm{N}$ & Y & Y \\
\hline Zhang and Chen, $2011^{31}$ & $\mathrm{~N}$ & $\mathrm{~N}$ & $\mathrm{~N}$ & Y & Y & Y \\
\hline $\mathrm{Li}, 2013^{32}$ & $\mathrm{~N}$ & Y & N & $\mathrm{N}$ & Y & N \\
\hline Liu et al, $2017^{33}$ & $\mathrm{~N}$ & Y & $\mathrm{N}$ & $\mathrm{N}$ & Y & Y \\
\hline Chen et al, $2015^{35}$ & $\mathrm{~N}$ & $\mathrm{~N}$ & N & Y & Y & Y \\
\hline Atkins et al, $2017^{36}$ & Y & $\mathrm{N}$ & $\mathrm{N}$ & Y & Y & Y \\
\hline Du et al, $2016^{38}$ & Y & Y & N & Y & Y & Y \\
\hline Li et al, $2012^{39}$ & Y & $\mathrm{N}$ & $\mathrm{N}$ & $\mathrm{N}$ & Y & N \\
\hline Cao et al, $2017^{43}$ & Y & Y & Y & Y & Y & Y \\
\hline Study design & & \multicolumn{5}{|c|}{ The study design was clearly described. } \\
\hline Inclusion criteria & & \multicolumn{5}{|c|}{ The inclusion or exclusion criteria of participants were clearly stated. } \\
\hline Validated measures & & \multicolumn{5}{|c|}{ Standardised questionnaire was used to measure medication adherence/non-adherence score. } \\
\hline Data analysis & & \multicolumn{5}{|c|}{ Statistical methods used to analyse the data were explicitly stated. } \\
\hline Reporting & & \multicolumn{5}{|c|}{ The findings of the study were objectively reported. } \\
\hline Content and utility & & \multicolumn{5}{|c|}{$\begin{array}{l}\text { Main findings, such as interpretations, inferences and themes, were synthesised based on evidence and were consistent } \\
\text { with the results. }\end{array}$} \\
\hline
\end{tabular}

N, no; NA, not applicable; Y, yes.

found that older patients have poor medication adherence, and the authors surmised that memory loss or inability to remember medication name, dosage, route and time may be a key explanatory factor. More recently, two studies ${ }^{17} 27$ found older age $(>60)$ to be a main influencing factor contributing to Chinese patients' poor adherence to cardioprotective medications.

\section{Gender (one article)}

Medication adherence can be different among male and female patients with CHD. Yang et $a l^{28}$ compared medication adherence between male and female patients with CHD and found that female participants had better adherence to aspirin, betablockers and ACE inhibitors/angiotensin II receptor blockers, while there was no significant difference between female and male participants in adherence to statins.

\section{Education (eight articles)}

Seven out of eight studies 316172027 29-31 revealed that a low educational level is a main influencing factor that contributes to poor medication adherence. Wang and $\mathrm{Li}^{16}$ investigated the reasons behind this relationship and found patients with a high educational level (> high school) are more likely to gather information about their medications; therefore, they have a better understanding and knowledge of statins. This better understanding contributed to better medication adherence. ${ }^{16}$ However, Jiang et al ${ }^{29}$ surveyed 5047 outpatients with stable angina from 298 hospitals in 15 Chinese provinces and found that patients with a high school or lower education level had higher beta-blockers adherence than those with a college degree. This result is different from our general finding that patients 
Table 3 Summary of studies included in review

\begin{tabular}{|c|c|c|c|c|}
\hline Author (year) & Research design & Sample & Location & Measuring medication adherence \\
\hline $\begin{array}{l}\text { Bi et al } \\
(2009)^{2}\end{array}$ & $\begin{array}{l}\text { A multicentre } \\
\text { prospective study }\end{array}$ & $\begin{array}{l}\mathrm{N}=2901 \text {, average age: } \\
64.5 \pm 12 \text {, female } 948\end{array}$ & $\begin{array}{l}51 \text { hospitals ( } 41 \text { tertiary and } 10 \\
\text { non-tertiary urban hospitals) } \\
\text { in China. }\end{array}$ & $\begin{array}{l}\text { Standardised paper case report forms were } \\
\text { used to document if participants were } \\
\text { adherent to their medication. }\end{array}$ \\
\hline $\begin{array}{l}\text { Jin et al } \\
(2014)^{3}\end{array}$ & $\begin{array}{l}\text { Two-group comparison } \\
\text { study }\end{array}$ & $\begin{array}{l}\mathrm{N}=469 \text {, female } 142 \\
(30.3 \%)\end{array}$ & Nanjing, Jiangsu Province, China. & Did not use instrument. \\
\hline $\begin{array}{l}\text { Zhang et al } \\
(2015)^{12}\end{array}$ & $\begin{array}{l}\text { Three-group } \\
\text { comparison study }\end{array}$ & $\begin{array}{l}\mathrm{N}=5926 \text {, female } 1095 \\
(18 \%)\end{array}$ & Beijing, China. & $\begin{array}{l}\text { Participants' medication adherence was } \\
\text { measured by their patterns of beta-blocker } \\
\text { use at hospital discharge and during the } \\
\text { first year after discharge: (1) always users, } \\
\text { (2) never users and (3) inconsistent users. }\end{array}$ \\
\hline $\begin{array}{l}\text { Jiang et al } \\
(2012)^{13}\end{array}$ & Cross-sectional study & $\begin{array}{l}864 \text { physicians } \\
\text { participated in the } \\
\text { survey, representing } \\
86 \% \text { of all attending } \\
\text { physicians and } \\
\text { residents in cardiology } \\
\text { departments in the } 35 \\
\text { hospitals. }\end{array}$ & $\begin{array}{l}35 \text { tertiary hospitals located in } \\
\text { urban areas of China. }\end{array}$ & Did not measure medication adherence. \\
\hline $\begin{array}{l}\text { Wang and Li } \\
(2014)^{16}\end{array}$ & $\begin{array}{l}\text { Retrospective analysis } \\
\text { study }\end{array}$ & $\begin{array}{l}\mathrm{N}=1368 \text {, age range: } \\
80-101 \text {, average age: } \\
86.4 \pm 6.6 \text {, female } 528\end{array}$ & Beijing, China. & Morisky-Green Questionnaire. \\
\hline Li $(2015)^{17}$ & $\begin{array}{l}\text { Retrospective analysis } \\
\text { study }\end{array}$ & $\begin{array}{l}N=138 \text {, age range: from } \\
54 \text { to } 76 \text {, average age: } \\
62.12 \pm 10.11 \text {, female } 68\end{array}$ & Enshi City, Hubei Province, China. & $\begin{array}{l}\text { Used a self-made scale. Very much like } \\
\text { MMAS-4, but one question is different. } \\
\text { Validity and reliability were not reported. }\end{array}$ \\
\hline
\end{tabular}

Findings

Theme topic

Medication adherence was high at the time Discharge, of hospital discharge, but decreased during lack of follow-up care. follow-up. Patients discharged on more drugs had even lower adherence.

Older patients had a significantly decreased Age, income, comorbidity, medication adherence than younger patients. adverse effects, education discharge, medication cost/insurance.

Consistent beta-blocker use after

Lack of follow-up care.

hospital discharge can lower mortality and cardiovascular events. Among 5926 participants with CHD, 2922 (49.3\%)

consistently used beta-blocker, whereas 1323

(22.3\%) never used it.

Medication adherence is related to

physicians. Knowledge of physicians was not precise or up-to-date.

Chinese people with CHD older than 80 years Age, education, patients' had poor adherence to statin. $22 \%$ of them lack of knowledge, did not adhere to their statins. income.

Adherence to statins is poor among Chinese Number of discharge people with CHD. $36.23 \%$ of them had poor medications, adherence.

adverse effects, income, patients' lack of knowledge, age, education, medication cost/insurance.

\begin{tabular}{|c|c|c|c|c|c|c|}
\hline $\begin{array}{l}\text { Ding et al } \\
(2013)^{* 18}\end{array}$ & Cross-sectional study & $\begin{array}{l}\mathrm{N}=903 \text {, average age: } \\
64.9 \pm 10.7, \text { female } 257 \\
(29.3 \%)\end{array}$ & Beijing, China. & Self-made questionnaire. & $\begin{array}{l}\text { LDL-C control rate is very low in people with } \\
\text { CHD living in Beijing. Only } 36.9 \% \text { reached } \\
\text { the standard of LDL-C control. }\end{array}$ & $\begin{array}{l}\text { Patients' lack of } \\
\text { knowledge, lack of follow- } \\
\text { up care. }\end{array}$ \\
\hline $\begin{array}{l}\text { Zhao et al } \\
(2015)^{19}\end{array}$ & Cross-sectional study & $\begin{array}{l}N=159 \text {, average age: } \\
61.7 \text { years, female } 58 \\
(36.5 \%)\end{array}$ & $\begin{array}{l}\text { Zhengzhou, Henan Province, } \\
\text { China. }\end{array}$ & MMAS-8. & $\begin{array}{l}\text { Among Chinese people with CHD, those who } \\
\text { lack knowledge about their medications } \\
\text { were more likely to divert from the } \\
\text { instructions for their medication. }\end{array}$ & $\begin{array}{l}\text { Patients' lack of } \\
\text { knowledge. }\end{array}$ \\
\hline Dai $(2013)^{\star 20}$ & Cross-sectional study & $\begin{array}{l}\mathrm{N}=200 \text {, age range: from } \\
28 \text { to } 67 \text {, female } 88\end{array}$ & $\begin{array}{l}\text { Donggu Village, Pinggu District, } \\
\text { Beijing, China. }\end{array}$ & $\begin{array}{l}\text { Used a self-made survey, which was tested } \\
\text { in a pilot study. }\end{array}$ & $\begin{array}{l}\text { Medication adherence is poor in Chinese } \\
\text { rural areas. } 61.7 \% \text { participants had poor } \\
\text { adherence. }\end{array}$ & $\begin{array}{l}\text { Number of discharge } \\
\text { medications, } \\
\text { adverse effects, patients' } \\
\text { lack of knowledge, } \\
\text { education. }\end{array}$ \\
\hline $\begin{array}{l}\text { Hu et al } \\
(2008)^{21}\end{array}$ & Cross-sectional study & $\begin{array}{l}N=4778 \text {, female } 1719 \\
(36.0 \%)\end{array}$ & $\begin{array}{l}52 \text { medical centres in } 6 \text { Chinese } \\
\text { cities: Shanghai, Beijing, } \\
\text { Guangzhou, Zhejiang, Tianjin and } \\
\text { Xinjiang. }\end{array}$ & $\begin{array}{l}\text { Did not use instrument. Adherence to statins } \\
\text { was measured as the rate of achieving the } \\
\text { target LDL-C level. }\end{array}$ & $\begin{array}{l}18.6 \% \text { of patients with CHD at high risk } \\
\text { of cardiac event were not taking statins as } \\
\text { lipid-lowering therapy; } 17.5 \% \text { of patients } \\
\text { with CHD at very high risk of cardiac event } \\
\text { were not taking statins. }\end{array}$ & Lack of follow-up care. \\
\hline $\begin{array}{l}\text { Jiang et al } \\
(2015)^{\star 29}\end{array}$ & Cross-sectional study & $\begin{array}{l}\mathrm{N}=5407 \text {, average age: } \\
67.6, \text { female } 2152 \\
(42.9 \%)\end{array}$ & $\begin{array}{l}298 \text { hospitals in } 15 \text { Chinese } \\
\text { provinces. }\end{array}$ & $\begin{array}{l}\text { Did not use instrument. Participants who } \\
\text { were adherent were defined as those who } \\
\text { were still on their medications at the third } \\
\text { month after baseline survey. }\end{array}$ & $\begin{array}{l}\text { Medication non-adherence is common in } \\
\text { Chinese people with CHD. Several factors } \\
\text { were identified. }\end{array}$ & Education. \\
\hline $\begin{array}{l}\text { Xu et al } \\
(2012)^{25}\end{array}$ & $\begin{array}{l}\text { A retrospective, cross- } \\
\text { sectional study }\end{array}$ & $\mathrm{N}=200$, female 62 & $\begin{array}{l}\text { Hangzhou, Zhejiang Province, } \\
\text { China. }\end{array}$ & $\begin{array}{l}\text { Medication use was calculated by } \\
\text { percentage of participants with CHD } \\
\text { who were prescribed cardioprotective } \\
\text { medication. }\end{array}$ & $\begin{array}{l}\text { Patients discharged from the Chinese } \\
\text { medical hospital were less likely to receive } \\
\text { angiotensin receptor blockers/ACEI and } \\
\text { beta-blockers than those discharged from the } \\
\text { general hospital. }\end{array}$ & Lack of follow-up care. \\
\hline $\begin{array}{l}\text { Li et al } \\
(2016)^{24}\end{array}$ & $\begin{array}{l}\text { A sequential cross- } \\
\text { sectional study }\end{array}$ & $\mathrm{N}=2463$, female 673 & $\begin{array}{l}\text { Hospitals were chosen from both } \\
\text { economic-geographical regions } \\
\text { (eastern, central and western) } \\
\text { and rural/urban regions. }\end{array}$ & $\begin{array}{l}\text { Did not use instrument. Used the } 2010 \\
\text { Chinese Guideline for Diagnosis and } \\
\text { Treatment of Patients with ST-Elevation } \\
\text { Myocardial Infarction to determine } \\
\text { whether patients received the appropriate } \\
\text { fibrinolytic dosage. Appropriate dosage } \\
\text { was defined as between } 80 \% \text { and } 120 \% \\
\text { of the recommendation. Underdosing was } \\
\text { defined as less than } 80 \% \text { and overdosing } \\
\text { was defined as more than } 120 \% \text { of the } \\
\text { recommended dose. }\end{array}$ & $\begin{array}{l}\text { Many patients with CHD were not treated } \\
\text { with fibrinolytic therapy: only } 49.5 \% \text { ideal } \\
\text { candidates for fibrinolytic therapy received it. }\end{array}$ & Lack of follow-up care. \\
\hline $\begin{array}{l}\text { Wang et al } \\
(2015)^{23}\end{array}$ & $\begin{array}{l}\text { Retrospective analysis } \\
\text { study }\end{array}$ & $\begin{array}{l}\mathrm{N}=2128, \text { female } 724 \\
(34.02 \%)\end{array}$ & $\begin{array}{l}20 \text { tertiary hospitals in } \\
\text { Heilongjiang Province of China. }\end{array}$ & $\begin{array}{l}\text { Did not use instrument. Medication } \\
\text { adherence rate was calculated as the } \\
\text { number of eligible patients who actually } \\
\text { received the recommended medication } \\
\text { divided by all patients eligible for it. }\end{array}$ & $\begin{array}{l}\text { The adherence rates to aspirin, beta-blocker } \\
\text { and thrombolytic were much less than the } \\
\text { target level recommended by the American } \\
\text { College of Cardiology/American Heart } \\
\text { Association as first-line treatments for } \\
\text { practice guidelines. }\end{array}$ & Lack of follow-up care. \\
\hline $\begin{array}{l}\text { Li et al } \\
(2009)^{22}\end{array}$ & Cross-sectional study & $\mathrm{N}=4778$, female 1719 & $\begin{array}{l}52 \text { centres in } 6 \text { cities in China: } \\
\text { Shanghai, Beijing, Guangzhou, } \\
\text { Xinjiang, Zhejiang and Tianjin. }\end{array}$ & Did not use instrument. & $\begin{array}{l}\text { The number of patients with CHD who } \\
\text { achieved the recommended LDL-C level was } \\
\text { suboptimal. } 18.6 \% \text { of patients with CHD } \\
\text { at high risk did not receive statin therapy; } \\
17.5 \% \text { at very high risk did not receive statin } \\
\text { therapy. }\end{array}$ & Lack of follow-up care. \\
\hline
\end{tabular}


Table 3 Continued

\begin{tabular}{|c|c|c|c|c|c|c|}
\hline Author (year) & Research design & Sample & Location & Measuring medication adherence & Findings & Theme topic \\
\hline $\begin{array}{l}\text { Li et al } \\
(2012)^{26}\end{array}$ & $\begin{array}{l}\text { Randomised controlled } \\
\text { trial }\end{array}$ & $\begin{array}{l}\mathrm{N}=16860 \text {, average age: } \\
63 \text {, female } 4384(26 \%)\end{array}$ & $\begin{array}{l}72 \text { study sites in } 14 \text { Chinese } \\
\text { cities. }\end{array}$ & $\begin{array}{l}\text { Did not use instrument. Medication } \\
\text { adherence was assessed by the rate of } \\
\text { medication usage among participants. }\end{array}$ & $\begin{array}{l}\text { Among Chinese people with CHD, } \\
\text { antiplatelet therapy was commonly used, but } \\
\text { the use of statins, beta-blockers and ACEls } \\
\text { was still not optimal. }\end{array}$ & $\begin{array}{l}\text { Age, comorbidity, } \\
\text { discharge, adverse effects. }\end{array}$ \\
\hline $\begin{array}{l}\text { Zhuang et al } \\
(2016)^{\star 27}\end{array}$ & Cross-sectional study & $\begin{array}{l}\mathrm{N}=348 \text {, age range: from } \\
45 \text { to } 75 \text {, average age: } \\
60 \pm 6 \text {, female } 166\end{array}$ & Shanghai, China. & $\begin{array}{l}\text { Did not use instrument. Medication } \\
\text { adherence was identified as following } \\
\text { physicians' prescriptions to take } \\
\text { antiplatelets. Non-adherence was identified } \\
\text { as changing dosage/frequency of prescribed } \\
\text { antiplatelets or stopping prescribed } \\
\text { antiplatelets. }\end{array}$ & $\begin{array}{l}\text { The percentages of participants who adhered } \\
\text { to their antiplatelets were } 98.3 \%, 92.8 \% \text { and } \\
81.9 \% \text {, respectively, in } 3 \text { months, } 6 \text { months } \\
\text { and } 12 \text { months after being discharged from } \\
\text { hospital. }\end{array}$ & Discharge, age, education. \\
\hline $\begin{array}{l}\text { Yang et al } \\
(2012)^{28}\end{array}$ & $\begin{array}{l}\text { Two-group comparison } \\
\text { study }\end{array}$ & $\begin{array}{l}\mathrm{N}=403 \text {, age range: } \\
48-84 \text {, average age: } \\
66.98 \pm 7.98 \text {, female } 161 \\
(40 \%)\end{array}$ & $\begin{array}{l}\text { Guiyang, Guizhou Province, } \\
\text { China. }\end{array}$ & $\begin{array}{l}\text { Morisky Medication Adherence } \\
\text { Questionnaire. }\end{array}$ & $\begin{array}{l}\text { Medication adherence can be different } \\
\text { among men and women. Female participants } \\
\text { had better medication adherence in aspirin, } \\
\text { beta-blockers and ACEl/angiotensin II } \\
\text { receptor blockers. There was no significant } \\
\text { difference between female and male } \\
\text { participants in adherence to statins. }\end{array}$ & Gender. \\
\hline $\begin{array}{l}\text { Huang et al } \\
(2014)^{\star 30}\end{array}$ & Longitudinal study & $\begin{array}{l}\mathrm{N}=262,51 \text { female } \\
(19.5 \%)\end{array}$ & $\begin{array}{l}\text { Nanning City, Guangxi Province, } \\
\text { China. }\end{array}$ & Morisky Green Levine Test. & $\begin{array}{l}6 \text { months after the } \mathrm{PCl}, 48.9 \% \text { of the } \\
\text { participants did not adhere to their } \\
\text { antiplatelets. } 12 \text { months after the } \mathrm{PCl} \text {, } \\
62.9 \% \text { participants did not adhere to their } \\
\text { antiplatelets. }\end{array}$ & $\begin{array}{l}\text { Income, education, } \\
\text { discharge, medication } \\
\text { cost/insurance. }\end{array}$ \\
\hline $\begin{array}{l}\text { Zhang } \\
\text { and Chen } \\
(2011)^{* 31}\end{array}$ & $\begin{array}{l}\text { Retrospective analysis } \\
\text { study }\end{array}$ & $\begin{array}{l}\mathrm{N}=500 \text {, average age: } \\
72 \pm 8.82 \text {, female } 228 \\
(45.6 \%)\end{array}$ & Chongqing, China. & $\begin{array}{l}\text { Did not use instrument. Participants were } \\
\text { interviewed within } 12 \text { months after } \mathrm{PCl} . \\
\text { If a participant told he/she has the same } \\
\text { medication as he/she has at the time of } \\
\text { discharge, the participant was regarded as } \\
\text { adherent. }\end{array}$ & $\begin{array}{l}\text { All participants adhered to their medications } \\
\text { at baseline. After being discharged from } \\
\text { hospital, the adherence rate started to } \\
\text { decrease. } 185 \text { ( } 37 \% \text { ) participants did not } \\
\text { adhere to their medications. Among the } \\
185 \text { participants, nearly } 97 \% \text { stopped their } \\
\text { medications } 6 \text { months later after being } \\
\text { discharged or even earlier. }\end{array}$ & $\begin{array}{l}\text { Comorbidity, discharge, } \\
\text { medication cost/insurance, } \\
\text { education. }\end{array}$ \\
\hline Li $(2013)^{\star 32}$ & Cross-sectional study & $\begin{array}{l}N=271 \text {, age range: from } \\
39 \text { to } 71 \text {, female } 100\end{array}$ & $\begin{array}{l}\text { Wuding County, Yunnan } \\
\text { Province, China. }\end{array}$ & $\begin{array}{l}\text { Did not use instrument. Participants were } \\
\text { identified as non-adherent as long as they } \\
\text { missed one dose, changed the dosage/ } \\
\text { frequency or made self-decision to stop } \\
\text { medication. }\end{array}$ & $\begin{array}{l}87.82 \% \text { participants did not adhere to } \\
\text { prescribed medications. Of these participants, } \\
22.9 \% \text { changed their medications' dosage or } \\
\text { frequency, } 30.6 \% \text { missed at least one dose } \\
\text { of medication, and } 34.3 \% \text { stopped physician- } \\
\text { prescribed medications and replaced them by } \\
\text { other medications. }\end{array}$ & $\begin{array}{l}\text { Income, } \\
\text { discharge. }\end{array}$ \\
\hline $\begin{array}{l}\text { Liu et al } \\
(2017)^{33}\end{array}$ & $\begin{array}{l}\text { An observational } \\
\text { cohort study }\end{array}$ & $\begin{array}{l}\mathrm{N}=404, \text { average age: } \\
58 \pm 10 \text {, female } 99 \\
(24.5 \%)\end{array}$ & Beijing, China. & $\begin{array}{l}\text { Did not use instrument. Medication } \\
\text { adherence was assessed by whether } \\
\text { participants stopped taking prescribed } \\
\text { medication—-ticagrelor. }\end{array}$ & $\begin{array}{l}39.1 \% \text { participants did not adhere to } \\
\text { their medications. Economic reasons and } \\
\text { haemorrhagic event were the main causes of } \\
\text { non-adherence to ticagrelor. }\end{array}$ & Adverse effects, income. \\
\hline $\begin{array}{l}\text { Chen et al } \\
(2015)^{35}\end{array}$ & Longitudinal study & $\mathrm{N}=512$, female 302 & Jiangsu Province, China. & $\begin{array}{l}\text { Did not use measurement. Medication } \\
\text { adherence was defined as taking the same } \\
\text { category of drugs at discharge during the } \\
\text { follow-up period. }\end{array}$ & $\begin{array}{l}\text { Compared with those with preserved left } \\
\text { ventricular ejection fraction (LVEF), patients } \\
\text { with reduced LVEF have lower medication } \\
\text { adherence. }\end{array}$ & Comorbidity. \\
\hline $\begin{array}{l}\text { Atkins et al } \\
(2017)^{36}\end{array}$ & A cohort analysis & $\begin{array}{l}\mathrm{N}=15140 \text {, female } 3651 \\
(24.11 \%)\end{array}$ & $\begin{array}{l}70 \text { hospitals from } 17 \text { provinces } \\
\text { of China. }\end{array}$ & $\begin{array}{l}\text { Did not use instrument. Medication } \\
\text { adherence was assessed by the rate of } \\
\text { medication usage among participants. }\end{array}$ & $\begin{array}{l}\text { Use of cardioprotective medications declines } \\
\text { over time after discharge. }\end{array}$ & Discharge. \\
\hline $\begin{array}{l}\text { Du et al } \\
(2016)^{38}\end{array}$ & $\begin{array}{l}\text { Randomised controlled } \\
\text { trial }\end{array}$ & $\mathrm{N}=964$, female 264 & $\begin{array}{l}\text { Zhengzhou, Henan Province, } \\
\text { China. }\end{array}$ & Did not mention. & $\begin{array}{l}\text { Intervention group had better medication } \\
\text { adherence than the control group. }\end{array}$ & Follow-up phone call. \\
\hline $\begin{array}{l}\text { Li et al } \\
(2012)^{39}\end{array}$ & $\begin{array}{l}\text { Randomised controlled } \\
\text { trial }\end{array}$ & $\mathrm{N}=100$, female 43 & Xi'an, Shaanxi Province, China. & $\begin{array}{l}\text { Proportion of days covered (PDC) was } \\
\text { used to measure medication adherence. } \\
\text { PDC=number of days when medications } \\
\text { were taken/number of days being } \\
\text { interviewed. }\end{array}$ & $\begin{array}{l}\text { Intervention group had significantly better } \\
\text { medication adherence than the control } \\
\text { group. }\end{array}$ & $\begin{array}{l}\text { Follow-up phone call, } \\
\text { medication memo card, } \\
\text { educational session/ } \\
\text { individual face-to-face } \\
\text { education. }\end{array}$ \\
\hline $\begin{array}{l}\text { Cao et al } \\
(2017)^{43}\end{array}$ & $\begin{array}{l}\text { Randomised controlled } \\
\text { trial }\end{array}$ & $\begin{array}{l}\mathrm{N}=236 \text {, average age: } \\
68.10, \text { female } 64 \\
(27.12 \%)\end{array}$ & $\begin{array}{l}\text { Chengdu, Sichuan Province, } \\
\text { China. }\end{array}$ & MMAS-8. & $\begin{array}{l}\text { Intervention group had significantly } \\
\text { higher medication adherence scores than } \\
\text { control group at } 30 \text { days and } 90 \text { days after } \\
\text { discharge. }\end{array}$ & $\begin{array}{l}\text { Educational session/ } \\
\text { individual face-to-face } \\
\text { education, follow-up } \\
\text { phone call. }\end{array}$ \\
\hline $\begin{array}{l}\text { Jiang et al } \\
(2007)^{40}\end{array}$ & $\begin{array}{l}\text { Randomised controlled } \\
\text { trial }\end{array}$ & $\mathrm{N}=167$, female 48 & $\begin{array}{l}\text { Chengdu, Sichuan Province, } \\
\text { China. }\end{array}$ & $\begin{array}{l}\text { The self-reported drug compliance scale, a } \\
5 \text {-point Likert scale ranging from } 1 \text { (totally } \\
\text { drug refusal) to } 5 \text { ( } 100 \% \text { drug compliance). } \\
\text { This instrument is reliable and valid. }\end{array}$ & $\begin{array}{l}\text { Medication adherence was decreased over } \\
\text { time, but to a significantly lesser extent in } \\
\text { intervention group. }\end{array}$ & $\begin{array}{l}\text { Educational session/ } \\
\text { individual face-to-face } \\
\text { education, booklet. }\end{array}$ \\
\hline $\begin{array}{l}\text { Zhao and } \\
\text { Wong } \\
(2009)^{44}\end{array}$ & $\begin{array}{l}\text { Randomised controlled } \\
\text { trial }\end{array}$ & $\begin{array}{l}N=200, \text { female } 53 \\
(53 \%)\end{array}$ & Tianjin, China. & $\begin{array}{l}\text { Did not mention the name of the survey, but } \\
\text { the authors explained the content validity of } \\
\text { the instrument was confirmed by experts in } \\
\text { the study team. }\end{array}$ & $\begin{array}{l}\text { Medication adherence was not significantly } \\
\text { different between the control group and } \\
\text { the intervention group at baseline, but the } \\
\text { intervention group participants' adherence } \\
\text { was better and became significant in weeks } \\
4 \text { and } 12 \text {. }\end{array}$ & $\begin{array}{l}\text { Educational session/ } \\
\text { individual face-to-face } \\
\text { education. }\end{array}$ \\
\hline $\begin{array}{l}\text { Zhao et al } \\
(2015)^{41}\end{array}$ & $\begin{array}{l}\text { Randomised controlled } \\
\text { trial }\end{array}$ & $\begin{array}{l}\mathrm{N}=90 \text {, did not mention } \\
\text { female }\end{array}$ & $\begin{array}{l}\text { Zhengzhou, Henan Province, } \\
\text { China. }\end{array}$ & $\begin{array}{l}\text { Questionnaire, did not mention the name of } \\
\text { the questionnaire, and did not mention its } \\
\text { reliability and validity. }\end{array}$ & $\begin{array}{l}\text { Medication adherence was significantly } \\
\text { better in the intervention group than in the } \\
\text { control group. }\end{array}$ & $\begin{array}{l}\text { Educational session/ } \\
\text { individual face-to-face } \\
\text { education, follow-up } \\
\text { phone call. }\end{array}$ \\
\hline $\begin{array}{l}\text { Wu et al } \\
(2012)^{45}\end{array}$ & $\begin{array}{l}\text { Two-group comparison } \\
\text { study }\end{array}$ & $\begin{array}{l}\mathrm{N}=110, \text { female } 22 \\
(20 \%)\end{array}$ & $\begin{array}{l}\text { Chengdu, Sichuan Province, } \\
\text { China. }\end{array}$ & $\begin{array}{l}\text { Did not use measurement. Interviewed } \\
\text { participants to see if they were still taking } \\
\text { their medication. }\end{array}$ & $\begin{array}{l}\text { Medication adherence of the intensive group } \\
\text { was much better than the control group. }\end{array}$ & Booklet. \\
\hline $\begin{array}{l}\text { Zhao et al } \\
(2015)^{42}\end{array}$ & $\begin{array}{l}\text { Randomised controlled } \\
\text { trial }\end{array}$ & $\mathrm{N}=120$, female 48 & $\begin{array}{l}\text { Zhengzhou, Henan Province, } \\
\text { China. }\end{array}$ & Did not use instrument. & $\begin{array}{l}\text { Intervention group had better medication } \\
\text { adherence than the control group. }\end{array}$ & $\begin{array}{l}\text { Booklet, educational } \\
\text { session/individual face-to- } \\
\text { face education, follow-up } \\
\text { phone call. }\end{array}$ \\
\hline
\end{tabular}




\begin{tabular}{llllll}
\hline Table 3 & Continued & & & \\
& & & \\
Author (year) & Research design & Sample & Location & Measuring medication adherence & Findings \\
\hline $\begin{array}{llll}\text { Fang and Li } \\
(2016)^{46}\end{array}$ & $\begin{array}{l}\text { Randomised controlled } \\
\text { trial }\end{array}$ & $\begin{array}{l}\mathrm{N}=280, \text { age range: } \\
38-69, \text { female } 80 \\
(28.58 \%)\end{array}$ & $\begin{array}{l}\text { Chengdu, Sichuan Province, } \\
\text { China. }\end{array}$ & MMAS-4. & $\begin{array}{l}\text { Participants in the intervention group } \\
\text { of using WeChat had better medication } \\
\text { adherence. }\end{array}$ \\
\hline
\end{tabular}

Articles marked with * were retrieved from Chinese database, the China National Knowledge Infrastructure. Articles in bold were written in Chinese.

ACEI, ACE inhibitor; CHD, coronary heart disease; LDL-C, low-density lipoprotein-cholesterol; MMAS-4, Four-ltem Morisky Medication Adherence Scale; MMAS-8, Eight-Item Morisky Medication Adherence Scale; PCI, percutaneous coronary intervention.

with a higher educational level are more likely to have better medication adherence.

\section{Income (six articles)}

Our review shows that low income was consistently found to be a factor related to medication non-adherence. ${ }^{3} \mathrm{Li}^{32}$ explored cardioprotective medication adherence among 271 patients with CHD and revealed that patients with high family income (> $¥ 50000$ per year) have significantly better medication adherence. Further, studies ${ }^{16} 17$ investigated patients with CHD on their adherence to statins and found that finances are an influencing factor to medication adherence. Studies ${ }^{30} 33$ on adherence to other cardioprotective medication such as antiplatelets also found the same result. Huang et $a l^{30}$ investigated the factors influencing medication adherence among 262 patients with CHD who received percutaneous coronary intervention and found that poor adherence to antiplatelets a year after hospital discharge was correlated with low economic status. Liu et $a l^{33}$ investigated the main cause of non-adherence to ticagrelor, an antiplatelet decreasing the risk of myocardial infarction, ${ }^{34}$ and found that low economic status is a main cause of non-adherence.

\section{Medication cost/insurance (four articles)}

Many studies s $^{173031}$ in our review found that high medication cost was related to poor medication adherence. For example, Zhang and $\mathrm{Chen}^{31}$ investigated 500 patients with CHD on their adherence to antiplatelet therapy and revealed that high medical payment was related to poor adherence. Specifically, patients having public medical insurance had higher medication adherence than patients who had rural cooperative medical care or needed to pay medical care out of own pocket. ${ }^{3031}$ Similarly, Jin $e t a l^{3}$ and $\mathrm{Li}^{17}$ found that medication cost was an influencing factor that contributed to poor adherence. Participants with no insurance or who had to pay medication out of pocket had poorer medication adherence. ${ }^{17}$

\section{Health status in relationship with adherence Comorbidity (four articles)}

Our review suggests that patients with CHD with comorbidity are more likely to have poor medication adherence. Zhang and Chen $^{31}$ studied 547 patients with CHD on their adherence to dual antiplatelet therapy 1 year after they underwent percutaneous coronary intervention and revealed that having comorbidities contributed to low medication adherence. Similarly, Chen et $a l^{35}$ studied the association between heart function and medication adherence and found that patients with CHD with reduced left ventricular ejection fraction had lower medication adherence. Furthermore, Jin et $a l^{3}$ found that the higher the number of comorbidities, the greater the chance of medication non-adherence. Li et $a l^{26}$ found that statin use was low among certain high-risk patients with CHD, including those with hypertension or diabetes, even though the benefits of statin are great among this population.

\section{Discharge (seven articles)}

Medication adherence among Chinese patients with CHD often drops significantly after discharge from the hospital. Zhuang et $\mathrm{al}^{27}$ studied 348 discharged patients with CHD about their adherence to antiplatelet therapy and revealed that medication adherence gradually decreased after patient discharge. Similarly, Li et $a l^{26}$ found that among 13150 patients with CHD, continued use of statin dropped substantially over time. Atkins et $a l^{36}$ also found that among 236 patients with CHD, continued use of cardioprotective medications declined over time. This result is similar to findings from two previous studies. ${ }^{32}$ The two studies found that recommended cardioprotective medications were commonly underused among Chinese patients with CHD one year after hospital discharge. To further investigate the relationship between discharge and medication adherence, Zhang and $\mathrm{Chen}^{31}$ and Huang $e t a l^{30}$ investigated the factors influencing medication adherence among patients with CHD who received percutaneous coronary intervention. They found the same results: adherence to antiplatelet dropped over time. ${ }^{3031}$ In summary, our review found that the longer the time after discharge, the poorer the medication adherence.

\section{Medication characteristics that are related to adherence Adverse effects (five articles)}

Adverse effects are associated with decreased medication adherence among patients with CHD. This was supported by several studies in our review. ${ }^{3} 17202633 \mathrm{Dai}^{20}$ surveyed 200 Chinese patients with CHD and found that the fear of adverse effects of cardioprotective medication was a barrier to adherence. Jin et $a l^{3}$ investigated the main influencing factors to medication non-adherence among patients with CHD and found the fear of adverse effects such as sexual dysfunction, depression and fatigue were a confirmed factor. Similarly, Liu et $a l^{33}$ investigated the main cause of the non-adherence to ticagrelor, an oral antiplatelet drug decreasing the risk of myocardial infarction, ${ }^{34}$ and found that adverse effect of ticagrelor, such as haemorrhage, is one main cause. $\mathrm{Li}^{17}$ studied 138 patients with $\mathrm{CHD}$ on their adherence to statins and found that adverse effects of statins are indeed a factor contributing to poor adherence. Taking statins over time can influence the musculoskeletal system, neurological system and liver dysfunction. ${ }^{17}$ To further study the association of adverse effects and non-adherence to statins, Li et $a l^{26}$ categorised 16860 patients into different risk levels. They found that statin use was low among high-risk patients with CHD even though the absolute benefits of using statins are high among this population. $\mathrm{Li}$ and colleagues thought this might be because of a treatment-risk paradox. Treatment-risk paradox is a phenomenon in which patients at high risk for adverse events receive less-intensive treatment than do patients at lower risk. ${ }^{37}$

\section{Number of discharge medications (two articles)}

Two studies in our review found that the greater the number of discharge medications patients received, the greater the chance of medication non-adherence. $\mathrm{Li}^{17}$ studied 138 patients 
with CHD on their adherence to statins and found that using multiple medications was an influencing factor that contributed to poor adherence. Also, Dai ${ }^{20}$ surveyed 200 patients with CHD living in a Chinese rural area and found that using multiple medications was a factor that contributed to poor medication adherence.

\section{Interventions to improve medication adherence vary in methods}

Our systematic review found that researchers explored various interventions to improve adherence to cardioprotective medication of patients with CHD. Follow-up phone calls, educational lectures, booklets and reminder cards were common methods found to be effective in improving medication adherence. Education and reminders are two key components of all interventions.

\section{Follow-up phone calls (five articles)}

Making follow-up phone calls to remind patients to take medications or give consultations on medication usage is the most common method we found in the review. Du $e^{2} a^{38}$ randomised 964 patients with CHD into an intervention or control group. Participants in the intervention group received medical consultations from a cardiologist through phone calls at 3, 6, 12 and 36 months postdischarge. The study found that the intervention group had significantly better medication adherence. ${ }^{38}$ Similarly, Li et $a l^{39}$ used phone call interviews to educate participants with myocardial infarction on relevant knowledge after discharge and found this intervention was effective in improving medication adherence. Telephone follow-up was used in many randomised controlled trials. ${ }^{40-42}$ For example, Jiang et al ${ }^{40}$ tested the effectiveness of a nurse-led cardiac rehabilitation educational programme in improving health behaviours among 167 randomly selected patients with CHD. In the programme, an experienced cardiac nurse provided patients with professional follow-up through telephone calls to monitor, facilitate and reinforce self-management practice of the patients. Patients who received the intervention demonstrated a significantly better performance in medication adherence. ${ }^{40}$ Similarly, Cao et $a l^{43}$ conducted a randomised controlled trial to test the effectiveness of a hospital-community partnership transitional programme on medication adherence. In the programme, family physicians and home nurses made structured telephone calls after patient discharge to reinforced health self-management behaviours and remind timely outpatient visits to hospital, whereas patients in the control group received routine care. Cao et $a l^{43}$ found that the intervention group had significantly higher medication adherence scores than the control group at 30 days and 90 days after discharge.

Zhao $e a^{41}$ examined the impact of using a clinical pharmacist support programme on medication adherence among patients receiving multidrug therapy for CHD in China. Compared with a usual care control group, the intervention group received pharmacist support that included medication review, patient education, lifestyle management, discharge guidance and telephone follow-up. Patients in the intervention group showed better medication adherence. ${ }^{41}$ Zhao and Wong ${ }^{44}$ tested the effectiveness of a nurse-led postdischarge transitional care programme for patients with CHD that included a community nurse who followed up with participants' adherence behaviour for 4 weeks. Compared with the control group, which received routine care, the intervention group had significantly better adherence to their medications. ${ }^{44}$
Booklet (three articles)

Giving a booklet for patients to refer important information of $\mathrm{CHD}$ and their medication was found to be effective in our review. However, none of the three studies used booklet alone; booklet was used with other interventions to improve medication adherence, such as follow-up phone calls, educational session or individual face-to-face education. Wu et $a l^{45}$ found patients in an intensive management group had better medication adherence than patients who received routine care. A booklet of CHD was provided to participants in the intensive management group. With such a booklet, patients can learn CHD-relevant knowledge from it. The booklet increased participants' awareness of taking medications. Similarly, Jiang $e t$ a $l^{40}$ provided a healthy heart booklet that covered medication management to patients with CHD to facilitate and reinforce their medication adherence. Zhao $e t \mathrm{al}^{42}$ investigated whether a pharmaceutical care intervention can improve medication adherence. In this intervention, an instructional list of each medication was provided to patients.

\section{Medication memo card (one article)}

A medication memo card was found to be an effective method to improve medication adherence. Li et $a^{39}$ conducted a randomised controlled trial to improve medication adherence of patients with CHD. Intervention in the study included giving a medication memo card to participants with a physician's prescription and time of taking medications. ${ }^{39}$ This method improved patients' medication adherence.

\section{Short message service (one article)}

Using a sample of 280 outpatients, Fang and $\mathrm{Li}^{46}$ examined the effectiveness of an electronic messaging support service programme as a means of providing discharged patients with reminders and coronary artery disease-related health information. Fang and $\mathrm{Li}^{46}$ found that compared with the control group who received phone calls, the group who received medication reminders via short message service (SMS) had better cumulative adherence to lipid-lowering therapy after 6 months of the programme.

\section{Mobile app (one article)}

Fang and $\mathrm{Li}^{46}$ built a public platform on one of the most popular mobile apps in China called WeChat. From a computer, healthcare providers regularly released to the WeChat platform CHD-related information on cardioprotective medication, including its function, method of use and side effects. Patients in the experimental group had open access to all information on the WeChat platform. ${ }^{46}$ Fang and $\mathrm{Li}^{46}$ found that compared with the control group, the experimental group had better cumulative adherence to lipid-lowering therapy after 6 months of the programme.

Educational session/individual face-to-face education (six articles) Jiang $e t a l^{40}$ tested the effectiveness of a nurse-led, hospital-initiated, cardiac rehabilitation educational programme to improve health behaviours of 167 patients with CHD, including medication adherence. The cardiac rehabilitation programme of this study was started in the hospital and maintained to 12 weeks after discharge, consisting of seven educational sessions covering (1) CHD and self-management principles, (2) medication management, (3) angina prevention and management, (4) physical exercise, (5) dietary management, (6) smoking cessation and (7) family support. Patients who received this 
intervention demonstrated a significantly better performance in medication adherence. ${ }^{40}$ In addition, educating participants' family members improved medication adherence. Li et $a l^{39}$ conducted a randomised controlled trial to improve medication adherence. Interventions for the experimental group included educating participants' family members on how to care for patients with myocardial infarction at months $1,3,6,9$ and 12 postdischarge. ${ }^{39}$ Individual face-to-face education provided by healthcare providers also can improve medication adherence. ${ }^{41}$ Zhao et $a l^{41}$ examined the impact of using a clinical pharmacist support programme on medication adherence among patients receiving multidrug therapy for CHD in China. Compared with a usual care control group, the intervention group received pharmacist support that included individual face-to-face patient education. As a result, patients in the intervention group showed significantly better understanding of the importance of medication adherence and improved medication adherence. ${ }^{41}$ Similarly, in the study conducted by Zhao et $a l^{42}$ Cao et al, ${ }^{43}$ and Zhao and Wong, ${ }^{44}$ healthcare providers gave medical directions and explained the importance of taking cardioprotective medications to patients. The result was that the medication adherence rate was higher in the pharmaceutical care group. ${ }^{42}$

In addition to investigating the advantages of each intervention, disadvantages of the interventions were rarely mentioned in the reviewed studies. Only one study ${ }^{46}$ discussed that mobile apps and SMS require patients to have access to a cellular data network; therefore, these interventions may be less applicable to patients in rural areas who do not have access to smart phones or cellular networks, or who are illiterate.

\section{DISCUSSION}

Cardioprotective medications were commonly prescribed as secondary prevention medication to patients with CHD in mainland China. ${ }^{2}$ In this systematic review, we found that adherence to these medications gradually decreased during a follow-up period. ${ }^{2} 1335$ This finding is a critical public health issue in China because long-term pharmaceutical therapy is essential for the treatment of CHD, and non-adherence to cardioprotective medications could render futile any effort to treat $\mathrm{CHD}^{47}$ and cause severe adverse health outcomes. In this review, we synthesised the findings to provide a clearer understanding of factors related to poor adherence, which we organised into several key themes. Also, we summarised all interventions that have been taken to improve medication adherence. The impact of the lack of medication-related knowledge among patients with CHD cannot be underemphasised and served as a barrier to medication adherence. Future programmes aimed to improve medication adherence should develop interventions to improve patients' knowledge of their medications, such as function, frequency and dosage. Our review showed that, in China, lack of proper follow-up care for patients with CHD regarding their medication-taking behaviours is also a barrier to medication adherence; therefore, to improve medication adherence, interventions should be taken to promote follow-up care. Future interventions aimed to improve medication adherence should increase access to care and care delivery of patients with CHD after their discharge from hospitals. This systematic review found that medication-taking reminders and educational materials were the two core components for successful interventions aimed to improve medication adherence. Therefore, future interventions should adopt these two core components.

Also, our review showed that physicians' prescribing practices can influence patients' medication-taking behaviours and health outcomes; therefore, factors that compromised physicians' prescribing practices should be studied. Several studies ${ }^{13} 2324$ in this review mentioned that physicians' knowledge deficiency of prevention guidelines was a factor. One possible reason for this knowledge deficiency could be the poor and slow dissemination of current guidelines. To improve this gap, a Chinese translation of current international guidelines could be helpful. Because most updated guidelines are made in the USA and Europe, they are written in English. Most Chinese physicians cannot read English or do not have time to read English guidelines. Finally, hospitals in mainland China should provide cardiologists, particularly young cardiologists, with further training opportunities on cardiology prevention and rehabilitation. Patients' lack of follow-up care may also be related to the healthcare system in China. The included studies did not provide direct evidence to support this point, but empirical evidence from other studies has shown that patients with CHD prefer to use hospitals rather than local primary healthcare clinics for treatment. ${ }^{48}$ Under the current system, most physicians in large hospitals are required to see a great number of patients each day, and they may not have a sufficient amount of time to provide advice to patients on medication adherence. Future studies should investigate the association of the number of patients seen by a physician on follow-up care and medication adherence.

We found that adherence is contingent on patients' health and demographic characteristics, including age, education, comorbidities, number of medications prescribed, adverse effects and insurance. This finding is consistent with those found in other countries. For example, Du $e t a l^{8}$ studied the impact of medication adherence on clinical outcomes through summarising studies conducted in Canada, USA, Argentina, Brazil, Italy, Paraguay and Spain. Du et al ${ }^{8}$ found that frequency, insurance coverage, patient education and follow-up were factors that can influence medication adherence. Similarly, $\mathrm{Ho}^{9}$ summarised studies from multiple nations and found that age, side effects, regimen complexity, low literacy and social support were related to medication adherence. Overall, patients from more affluent backgrounds and with access to care were likely to have better medication adherence. Many interventions have been tested by researchers to improve medication adherence. Unimodal interventions were less successful than multimodal interventions because medication non-adherence was often related to multiple factors. ${ }^{9}$ In our systematic review, we found a similar result that interventions to improve medication adherence varied in methods, but that education and reminder were the consistent key components of all interventions.

We used a set of criteria to assess the quality of evidence provided by each of the included 33 studies. These criteria included study design, sample selection, measures, statistical analyses, reporting, content and utility. ${ }^{49}$ Our review revealed a significant variation in the quality of reviewed studies. Overall, studies published in English journals provided more methodological details than those published in Chinese journals. The major limitations to some of the 33 studies included using non-representative samples, unstandardised assessment measures and an insufficient number of longitudinal findings. Therefore, findings from this review are not conclusive.

However, they provide a foundation from which to conduct future research. In particular, findings suggest the following: (1) More research should be conducted on how to establish high-quality health educational programmes aimed to increase patients' medication adherence. (2) In China, educating patients to take medications is the responsibility of healthcare 
providers. However, this review found that many Chinese physicians did not provide patients advice on the importance of medication adherence. This might be due to physicians' insufficient or not up-to-date knowledge of cardioprotective medications or due to physicians' lack of time allocated for each patient. Further, no articles in this review explored nurses' and pharmacists' knowledge of cardioprotective medications. To further explore the reasons for poor cardioprotective medication adherence among patients with CHD in mainland China, future studies should focus on the roles of all healthcare providers on patient education and prescribing patterns. Improving discharge prescription of cardioprotective medications and promoting long-term patient adherence are critically needed in China. (3) Many studies in this review were conducted in urban areas of China. Given that there is a big difference in medical care between urban areas and rural areas of China, future research should study medication adherence among patients with CHD living in rural China. (4) In the meantime, income and cost are consistently found to be associated with medication adherence. China is in the process of undergoing healthcare reform and extending health insurance coverage in rural areas. Demonstration projects are needed to examine the cost-effectiveness of health insurance extension on medication adherence. More robust studies are needed to examine the mediator and moderator factors associated with medication adherence using longitudinal data.

Acknowledgements We thank Jamie Conklin, Research and Education Librarian at Duke University Medical Center Library, for assistance in building the combination of index and search terms.

Contributors All authors contributed to the conception and design of the study. ZN, LD and Dr. Ryan Shaw were responsible for the data acquisition and analysis. All authors contributed to data interpretation and to writing and approving the final manuscript.

Funding The authors have not declared a specific grant for this research from any funding agency in the public, commercial or not-for-profit sectors.

Competing interests None declared.

Patient consent for publication Not required.

Provenance and peer review Not commissioned; externally peer reviewed.

\section{REFERENCES}

1. Kolandaivelu K, Leiden BB, O'Gara PT, et al. Non-adherence to cardiovascular medications. Eur Heart J 2014;35:3267-76.

2. Bi Y, Gao R, Patel A, et al. Evidence-based medication use among Chinese patients with acute coronary syndromes at the time of hospital discharge and 1 year after hospitalization: results from the clinical pathways for acute coronary syndromes in China (cPACs) study. Am Heart J 2009;157:509-16.

3. Jin $\mathrm{H}$, Tang C, Wei Q, et al. Age-related differences in factors associated with the underuse of recommended medications in acute coronary syndrome patients at least one year after hospital discharge. BMC Cardiovasc Disord 2014;14.

4. Gong P, Liang S, Carlton EJ, et al. Urbanisation and health in China. The Lancet 2012;379:843-52 https://doi.org/

5. World Health Organization. China: who statistical profile, 2015. Available: http:// www.who.int/gho/countries/chn.pdf?ua=1 [Accessed 29 Oct 2017].

6. Zhang X-H, Lu ZL, Liu L. Coronary heart disease in China. Heart 2008;94:1126-31.

7. National Institutes of Health. How to prevent and control coronary heart disease risk factors, 2016. Available: https://www.nhlbi.nih.gov/health/healthtopics/topics/hd/ prevent\#Medicines [Accessed 29 Oct 2017].

8. Du L, Cheng Z, Zhang Y, et al. The impact of medication adherence on clinical outcomes of coronary artery disease: a meta-analysis. The American Journal of Medicine 2017;24:962-70.

9. Ho PM, Bryson CL, Rumsfeld JS. Medication adherence: its importance in cardiovascular outcomes. Circulation 2009;119:3028-35.

10. Hamm CW, Bassand JP, Agewall S, et al. ESC guidelines for the management of acute coronary syndromes in patients presenting without persistent ST-segment elevation: the task Force for the management of acute coronary syndromes (ACS) in patients presenting without persistent ST-segment elevation of the European Society of cardiology (ESC). European Heart Journal 2011;32:2999-3054.
11. Menzin J, Wygant G, Hauch O, et al. One-year costs of ischemic heart disease among patients with acute coronary syndromes: findings from a multi-employer claims database. Current Medical Research and Opinion 2008;24:461-8.

12. Zhang $H$, Yuan $X$, Zhang $H$, et al. Efficacy of long-term $\beta$-blocker therapy for secondary prevention of long-term outcomes after coronary artery bypass grafting surgery. Circulation 2015;131:2194-201.

13. Jiang J, Hong T, Yu R, et al. Knowledge of secondary prevention guidelines for coronary heart disease: results from a physicians' survey in China. European Journal of Preventive Cardiology 2012;19:991-8.

14. Martin LR, Williams SL, Haskard KB, et al. The challenge of patient adherence. Therapeutics and Clinical Risk Management 2005;1:189-99.

15. Jimmy B, Jose J. Patient medication adherence: measures in daily practice. Oman Medical Journal 2011;26:155-9.

16. Wang K. Li X: 80 岁以上老年 心病患者他汀类药物依从性分析 [Compliance of Statins in Elderly Patients Aged over 80 Years with Coronary Heart Disease]. China Pharmaceuticals 2014;23:37-8.

17. LiW. 他汀类药物在 心病二级预防中应用依从性相关 病例对照 究 [A comparison study of factors related to adherence to statins in the secondary prevention of coronary heart disease]. Chinese Journal of Integrative Medicine on Cardio- /Cerebrovascular Disease 2015;13:680-1.

18. Ding RJ, CS M, Chen H, et al. 北京地区心内科门诊 心病患者胆固醇控制 现状调 查 [Control rate of increased low-density lipoprotein cholesterol levels in cardiology outpatients with coronary heart disease in Beijing]. Zhonghua Xin Xue Guan Bing Za Zhi 2013;41:251-5.

19. Zhao $\mathrm{S}$, Zhao $\mathrm{H}$, Wang $\mathrm{L}$, et al. Education is critical for medication adherence in patients with coronary heart disease. Acta Cardiologica 2015;70:197-204.

20. Dai H. 农村 心病二级预防调脂治疗的依从性调查 [Investigation of the compliance of secondary prevention and lipid-lowering treatment therapy in rural patients with coronary heart disease].. Chinese Journal of Clinical Rational Drug Use 2013;6.

21. Hu D, Li J, Li X, et al. Investigation of blood lipid levels and statin interventions in outpatients with coronary heart disease in China. Circulation Journal 2008;72:2040-5

22. Li X, Xu Y, Li J, et al. The gender differences in baseline characteristics and statin intervention among outpatients with coronary heart disease in China: the China cholesterol education program. Clinical Cardiology 2009;32:308-14.

23. Wang Y, Fu R, Wang Z, et al. Assessing the quality of care for patients with acute myocardial infarction in China. Clinical Cardiology 2015;38:327-32.

24. Li J Li X Ross JS, et al. Fibrinolytic therapy in hospitals without percutaneous coronary intervention capabilities in China from 2001 to 2011: China PEACE-retrospective AMI study. European Heart Journal: Acute Cardiovascular Care 2016;6:232-43.

25. HM X, Cai HW, Dai HB, et al. Use of evidence-based pharmacotherapy for secondary prevention of coronary heart disease: a Chinese medicine Hospital versus a general Hospital. Chinese Journal of Integrative Medicine 2012:20:375-80.

26. Li J, Chen YP, Li X, et al. Use of secondary preventive medications in patients with atherosclerotic disease in urban China: a cross-sectional study of 16,860 patients. Chinese Medical Journal 2012;125:4361-7.

27. Zhuang G, Ding B. Huang X: 出院 心病患者抗血小板治疗依从状况及影响 [Status of Compliance to Antiplatelet Therapy in Discharged Patients with Coronary Heart Disease and Analysis on the Influencing Factors]. Medical Recapitulate 2016;22:2394-6.

28. Yang J, Li Y, Yuan W, et al. 性别对PCI术后患者服药依从性和生活质量影响的 究 [The effect of gender on medication adherence and quality of life of patients after percutaneous coronary intervention]. Journal of Tropical Medicine 2012;12:610-2.

29. Jiang J, Wang $X$, Jia J, et al. Current status and influence factors of beta blocker prescription in Chinese patients with stable angina pectoris]. Zhonghua Xin Xue Guan Bing Za Zhi 2015;43:227-33.

30. Huang H, Ying Y, Li W, et al. 脉血运重建术后抗血小板药物依从性影响 分 析 [Analysis of factors influencing anti-platelet drug compliance after coronary revascularization]. Guangxi Medical Journal 2014;36:1612-4.

31. Zhang Y, Chen Q. PCI术后 1 年患者双联抗血小板药物治疗依从性随访 究 [Adherence with dual antiplatelet therapy and its influence on clinical outcomes in patients after undergoing percutaneous coronary stenting for 1 year]. Journal of Chongqing Medical University 2011;36:503-6.

32. Li Y. 心病二级预防用药依从性及其影响 探析 [Exploration of medication adherence in the secondary prevention of coronary heart disease and its influencing factors]. Xin Xue Guan Bing Fang Zhi Zhi Shi 2013;2:7-9.

33. Liu J, Yan HB, Song L, et al. 急性 状动脉综合征患者院外服用替 瑞洛现状调 查分析 [Contemporary use of ticagrelor in patients with acute coronary syndrome after discharge]. Zhonghua Yi Xue Za Zhi 2017:97:1165-9.

34. National Library of Medicine. Ticagrelor, 2017. Available: https://livertox.nlm.nih.gov/ Ticagrelor.htm [Accessed 30 Oct 2017].

35. Chen Z, Ding Z, Wang $X$, et al. Reduced heart function predicts drug-taking compliance and two-year prognosis in Chinese patients with stable premature coronary artery disease. J Clin Med Res 2015:7:154-60.

36. Atkins ER, Du X, Wu Y, et al. Use of cardiovascular prevention treatments after acute coronary syndrome in China and associated factors. International Journal of Cardiology 2017;241:444-9 
37. DT K, Mamdani M. Alter dA: lipid-lowering therapy with statins in high-risk elderly patients: the Treatment-Risk paradox. JAMA 2004;291:1864-70.

38. Du L, Dong P, Jia J, et al. Impacts of intensive follow-up on the long-term prognosis of percutaneous coronary intervention in acute coronary syndrome patients - a single center prospective randomized controlled study in a Chinese population. European Journal of Preventive Cardiology 2016;23:1077-85.

39. JX L, Li Y, Yang L, et al. Study on the effect of Multi-factorid optimized holistic nursing on medication adherence and cardiovascular events of patients with Myocarid infarction. Progress in Modern Biomedicine 2012;12:6714-6.

40. Jiang X, Sit JW, Wong TKS. A nurse-led cardiac rehabilitation programme improves health behaviours and cardiac physiological risk parameters: evidence from Chengdu, China. Journal of Clinical Nursing 2007;16:1886-97.

41. Zhao SJ, Zhao HW, Du S, et al. The impact of clinical pharmacist support on patients receiving multi-drug therapy for coronary heart disease in China. Indian Journal of Pharmaceutical Sciences 2015;77:306-11.

42. Zhao SJ, Zhao HW, Du S, et al. 药学服务对接受多药治疗的 心病病人预后 的影响 [Impact of pharmaceutical care on the prognosis of patients with coronary heart disease receiving multidrug therapy]. Pharmaceutical Care and Research 2015;15:179-81.

43. Cao X-Y, Tian L, Chen L, et al. Effects of a hospital-community partnership transitional program in patients with coronary heart disease in Chengdu, China: a randomized controlled trial. Japan Journal of Nursing Science 2017;14:320-31.
44. Zhao Y, Wong FKY. Effects of a postdischarge transitional care programme for patients with coronary heart disease in China: a randomised controlled trial. Journal of Clinical Nursing 2009;18:2444-55.

45. JHW, Sun CL, Zhang SM, et al. 门诊规范化管理对老年 心病患者预后的影响 [Effects of normalization management on prognosis in elderly patients with coronary artery disease]. Chinese Journal of Evidence-Based Medicine 2012;12:520-3.

46. Fang R, Li X. Electronic messaging support service programs improve adherence to lipid-lowering therapy among outpatients with coronary artery disease: an exploratory randomised control study. Journal of Clinical Nursing 2016;25:664-71.

47. World Health Organization. Chronic diseases and health promotion, 2003. Available: http://www.who.int/chp/knowledge/publications/adherence_report/en/ [Accessed Retrieved 1 Feb 2019].

48. Ni Z, Liu C, Wu B, et al. An mHealth intervention to improve medication adherence among patients with coronary heart disease in China: development of an intervention. International Journal of Nursing Sciences 2018;5:322-30.

49. Wu B, Fillenbaum GG, Plassman BL, et al. Association between oral health and cognitive status: a systematic review. J Am Geriatr Soc 2016:64:739-51.

50. Moher D, Liberati A, Tetzlaff J, et al. Preferred reporting items for systematic reviews and meta-analyses: the PRISMA statement. Ann Intern Med 2009;151:264-9. 